\title{
Pandémie zombie Monstres et mystères de Luis Felipe Fabre
}

\author{
Nathalie Galland Boudon \\ Université de Bourgogne, MCF \\ nathalieboudon@hotmail.com
}

Bajo las matas
En los pajonales
Sobre los puentes
En los canales
Hay Cadáveres

Néstor Perlongher

«What is poetry ?» se demandait autrefois Nicanor Parra... Qu'est-ce qu'écrire de la poésie aujourd'hui au Mexique $?^{1}$ semble suggérer Luis Felipe Fabre. Avec Poemas de terror y de misterio (Almadía, 2013), quelque chose dérape et fait glisser au-delà de lui-même le champ de la poésie : alors sorte de terrain vague volontiers nocturne où se retrouve l'écriture poétique, tendue par les motifs du roman noir ${ }^{2}$, entre fait divers racoleur et épisode de série B. Là, le lieu du poème est un terreau cauchemardesque où surgissent morts vivants et autres visages post-humains dans le rectangle de la page, qui est aussi celui de la pellicule filmique souvent convoquée, ou du trou en terre. Bref : un recueil qui tantôt s'égraine à tombeau ouvert, tantôt dispose un lent cortège funèbre de poèmes comme des corps planant, rôdant, se traînant, aux voix qui grincent ou qui chuchotent.

Si le titre du recueil dit déjà quelque chose d'une conception ludique ou transgressive de la poésie, le texte fait la part belle à un projet poétique qui désoriente, comme en leur temps les antipoemas de Parra secouaient tout à la fois policía et poesía ${ }^{3}$. En ouvrant la page aux frictions du réel, au trivial, à l'incongru, Luis Felipe Fabre pousse à son comble la désacralisation d'une ars poética victime d'un certain académisme ou de la déploration consensuelle de la violence extrême. Contrepoint d'humour noir, le recueil pour autant ne se résout pas dans la forme de la parodie ou de l'imitation esthétique du sous-genre littéraire ou cinématographique, mais questionne véritablement le sens de la poésie contemporaine en prise avec l'objet dégradé du monde : poétiquement incorrect, impur (et visant sans aucun doute un lectorat plus large), exquisément cadavérique, le recueil se déploie, redistribue dans ses poèmes les clairs-obscurs d'un Mexique de catacombes. Le texte montre, pareille à une succursale tropicale de films gore hollywoodiens, à un hypermarché des horreurs où déambuleraient côte à côte toutes les figures du monstre, de Dracula à Frankenstein et sa fiancée, de la momie sur le retour au loup-garou, à une énigmatique Sor Juana transfigurable, tous ex-humains démultipliés, autant de figures d'ombres portées au seuil du texte et qui viennent concrètement ponctuer les six sections poétiques du recueil. Le texte montre, mais ne coagule pas, comme cette foule de zombies précipitée dans ses pages refuse à son tour de se résorber. Il montre et il cache, jouant en parallèle sur le mystère, l'ésotérique, l'invisible, le non sens, autres faces de l'opacité du monde.

1 Au cours des huit dernières années, de 60000 à 80000 personnes ont perdu la vie au Mexique, dans un contexte de violence extrême, de " guerre » initiée contre le narcotrafic par le gouvernement de F. Calderón. S'ajoutent à ces morts, dans la filiation d'Acteal et de bien d'autres massacres, les 43 étudiants disparus d'Ayotzinapa, Gro, en septembre dernier, disparitions terrifiantes et exemplaires du fonctionnement d'un nécro-pouvoir.

2 On pourrait évoquer le recueil Muerte en la rua Augusta, de Tedi López Mills, Oaxaca : Almadía, 2009.

3 Nicanor Parra, Poemas parfa desorientar a la policía poesía, Madrid : Visor, 2006. 
Véritable archéologie de la peur intime qui fouille les possibilités du texte poétique à ses marges, le recueil met en branle un texte-performance qui dit l'épouvante face à une irrépressible contagion de l'enfer, une pandémie de mort sans cesse alimentée par le terrorisme du narcotrafic et de l'état réunis dans ce Mexique contemporain.

Nous interrogerons d'abord ici cette puissance de contagion de la terreur, rendue par des mots qui suivent eux-mêmes un processus de transmission, formant avec d'autres représentations esthétiques une chaîne causale où la monstruosité devient spéculaire. La terreur alors se vit, se pense, s'érige à la fois comme fond synesthésique et foyer de contagion gagnant tout l'espace, espace intime ou tissu social déjà en décomposition. C'est que le Mexique a cédé face à la pandémie zombie : contagion du corps, du regard, de la sensation, de l'imaginaire. Les hantises du présent, de la solitude, de la corruption, du chaos rayonnent en tout lieu et font de la figure monstrueuse la seule latence du monde. Précisément, nous explorerons dans un deuxième temps cette catastrophe zombie déployée par le recueil, qui dit un réel devenu proie d'un pandémonium global, espace d'expérience de la monstruosité généralisée. Enfin, nous nous attacherons à saisir les pages ellesmêmes comme espace ultime de la contagion : le monstre devenu écriture, excavation-palimpseste, réservoir d'une nécro-poésie où les mo(r)ts reviennent demander leur reste.

\section{Hantises du présent}

Il est intéressant de suivre, notamment dans les deux premières sections du recueil respectivement intitulées «Avances » et « Breve registro de algunos eventos artísticos y otras experiencias escalofriantes $»$, des poèmes qui sont des traces, des choix, qui disent des influences. Les «Trailer 1 » et «Trailer 2 », comme des bandes-annonces, posent véritablement les termes d'une écriture poétique qui change de focalisation et incorpore le marketing et les codes de la postproduction cinématographique. Là, les vers sont des images, pitchs et clips à la fois, avec effet d'ambiance thriller, et apparaissent dans des formes accidentées ou accidentelles - écarts, césures arbitraires, isolement -, projettent des fragments d'altérité qui se reproduisent. Quand l'accumulation anaphorique dit déjà la contagion, contagion de l'incertain en suspens dans le blanc :

\footnotetext{
Una chica desaparece en circunstancias misteriosas: otra

C'est une manière, inaugurale, légère et dévastatrice à la fois, de renseigner le lecteur sur le monde à partir de la peur et des figures monstrueuses qui le façonnent : ici « una noche, un cuello, un alarido, unos colmillos », là une « estampida de murciélagos $»^{5}$, tous les clichés de la série B mis

4 Luis Felipe Fabre, Poemas de terror y de misterio, Oaxaca : Almadía, 2013, p. 14.

5 Idem, p. 13 pour les deux citations. 
en vers, en gros plans, et servis par une ponctuation démonstrative («:») qui devient émotionnelle à force de systématicité. Le dispositif poétique crée d'emblée de l'instabilité à partir de la dissonance anaphorique des figures monstrueuses : « las chicas han vuelto » et précisément c'est bien ce volver polymorphe qui établira l'enjeu du recueil. Écrire aujourd'hui au Mexique, c'est peut-être revenir au réel le plus cru, contre l'état, contre l'oubli, contre la violence du terrorisme narco. Aussi le lieu du poème incarne-t-il cet espace du retour (transfiguration, métamorphose, combat), retour de l'expérience, surface-écran, scène de projection des figures classiques de la terreur, étrange salle de cinéma où peuvent aussi se rejouer les mystères surréalistes, poétiques, romantiques, mythologiques, ésotériques.

Terreur et mystère ouvrent bien le recueil, mais c'est la performance qui va mobiliser une écriture poétique « breve registro », espace dépositaire, témoin expérimental. De fait les six poèmes numérotés de la section - clairement structurés par l'impact de performances explorant la peur et menées dans le cadre de Proyecto líquido ${ }^{6}$ incarnent ce passage du corps à la lettre. Viennent se loger dans les vers les sensations corporelles, mentales du spectateur-participant à la performance : déambulations aléatoires dans l'obscurité, peur du noir, mutilations des sens, notamment de la vue, crissements, présences diffuses, tensions multiples de l'inconnu. La performance donnée en public trouve sur la page un lieu où s'étirer, où prolonger l'expérience individuelle pour dire du collectif, en écho intime à la douleur d'une société, d'un monde devenu immonde. L'écriture accueille la peur, la refait vibrer sur une page membrane où opèrent les désarticulations de langage et d'humanité : isolement, brèches, souffles coupés ou retenus, équilibres en suspens, solitudes miroitantes, sphères d'angoisses qui dérèglent la communication avec le monde... S'appropriant les morts devenus légions, les nouvelles nécropoles ouvertes, la terreur alors infuse le visible et l'imaginaire, présence mondaine et représentations : la pandémie zombie, c'est d'abord la contagion de la terreur, sa mise en abîme dans le texte.

Le premier poème «1 / Caminar con los ojos cerrados », dédié à Felipe Zúniga, revit littéralement la performance de Meiro Koizumi : Another vision ${ }^{7}$, avant de la mettre à distance :

\author{
Caminar con los ojos cerrados. Avanzar \\ a tientas. Los ojos \\ cerrados. La boca cerrada: sellada \\ con cinta adhesiva. Avanzar. Ir \\ a ciegas. Caminar con los ojos cerrados \\ por una casa deshabitada. Tropezar. Mantener los \\ ojos \\ cerrados por instrucciones de un artista. Tropezar8
}

C'est par la fragmentation des unités sémantiques que s'impose la figure du monstre : infinitifs et substantifs à l'arrêt ; mots en équilibre, « ojos » justement, qui forme un visage ébahi, les o grands ouverts, rayonnant dans la solitude. Clé d'appréhension de l'activité artistique, la peur joue précisément sur l'impossibilité du voir, sur un regard condamné à une autre palpation, interne,

6 Dans le cadre du Proyecto líquido, cycle de performances organisé par le collectif Fundación Alumnos 47 à Mexico en juin 2012 sur le thème de la peur, 11 artistes performers ont été réunis ainsi que 9 auteurs, narrateurs et poètes, conviés à rendre compte de leur expérience des performances. Le projet a donné naissance à un blog du même nom : Proyecto líquido, (para hablar sobre el miedo que vivimos en México) Site consultable sur proyectoliquido2012.wordpress.com

7 Le travail de création de Meiro Koizumi est consultable sur http://www.meirokoizumi.com/. Sa performance, réalisée pour Proyecto líquido, sur http://proyectoliquido2012.wordpress.com/2012/06/18/meiro-kozumi/ ou sur http://www.meirokoizumi.com/main $\% 20$ page $\% 20$ frame.html

8 Huit premiers vers de « 1 / Caminar con los ojos cerrados », Luis Felipe Fabre, idem, p. 21. 
mémorielle. L'absence comme première figure de la monstruosité, comme métaphore ; la présence menaçante, impalpable, projetée. Le poème recrée l'exploration d'un territoire où le corps souffre, se perd, s'interroge, ne reconnaît pas, ne se reconnaît pas. Et c'est par une marche forcée des mêmes mots, des mêmes actions sans cesse réitérées dans le vers et sur la page, que le poème avance à coup de «tropezar ", de " caminar », exorbités. Les mots paraissent désorientés, bousculés dans l'espace aveuglé de la page où l'éclat du blanc dit paradoxalement l'obscurité de la pièce vide, le même espace de liquidation des repères, géographiques et géo-poétiques.

La tonalité de la performance est évidente : le lecteur-spectateur est l'observateur-participant d'une scène d'épouvante qui serait aussi le jeu de l'art. Les mots redisposent la théâtralité de la performance, qui est aussi scénario de la séquestration, du corps réduit à la nuit, entravé, errant entre des murs abandonnés. L'artiste alors devient preneur d'otage, le poète preneur de son intime plus que de voix, dérobant toute extériorité. Il ne reste alors que le corps et la sensation pour éprouver et dire l'effet de la peur à l'intérieur de nous. C'est que l'écriture poétique assume sans doute aussi le postulat de la nécessité d'un partage de l'action, la nécessité qu'il y a à faire de son corps le lieu d'un ressenti intime à partir duquel pourra se construire le sens, fidèle en cela au spectateur émancipé de Jacques Rancière :

Les spectateurs voient, ressentent et comprennent quelque chose pour autant qu'ils composent leur propre poème, comme le font à leur manière acteurs ou dramaturges, metteurs en scène, danseurs ou performers 9 .

Cette première section poétique projette de l'ombre, de l'opaque, du sensible, fait palpiter des visions aussi - le corps de pianos mécaniques pendant des arbres, appel à d'autres corps silenciés, « 2 / Pianolas en los árboles,... $»^{10}-$, visions qui déroutent, inquiètent et propulsent paniques et questionnements sans réponses :

¿Viniste al performance de VestAndPage? ¿Viniste al performance de Yoshua Okón?

¿Y a lo de Galia Eibenschutz? ¿Viniste? ¿Te fuiste?

¿Huiste? ¿Llegaste? ¿Adónde? ¿Cómo? ¿Qué

fue ese ruido? ¿Viniste? ¿Y a lo de Marcela Armas

y Gilberto Esparza? ¿Por qué? ¿Qué?

¿Cómo? ¿A dónde? ¿Qué fue ese ruido? ¿Lo

escuchaste?

¿Qué me decías? ¿Una silla? ¿Una silla vacía?

¿Nadie? ¿Ninguno? ¿No has venido? ¿Estás ahí?

¿Viniste?

¿Y a lo de Pedro Reyes? ¿Te gustó? ¿Y lo de Carlos

Amorales?

¿No pudiste? ¿Miedo? ¿Yo? ¿Yo qué? ¿Qué? ¿Qué

fue ese ruido?

¿Vidrios rotos? ¿Pájaros negros? ¿No? ¿No te gustó?

¿No te pareció pertinente? ¿Ahora? ¿En México?

$¿$ Te has fijado que ya todos los poetas mexicanos

tienen su poemita sobre la violencia? ¿No? ¿No

lees poesía? ¿Qué no qué? ¿Qué me decías? ¿Qué fue ese ruido?

¿Una mariposa negra? ¿El timbre? ¿A estas horas?

¿Cómo? ¿Qué?

¿Qué ahí estaba quién? ¿Dónde? ¿Un video? ¿Una

proyección?

¿De verdad? ¿Y cómo estuvo? ¿No fuiste? ¿No? ¿Cuál?

9 Jacques Rancière, Le spectateur émancipé, Paris : La Fabrique Éditions, 2008, p. 22.

10 Un poème de Luis Felipe Fabre, op. cit., p. 23, en correspondance avec la performance Matanga, pianolas en los árboles, de Carlos Amorales. Voir https://proyectoliquido2012.files.wordpress.com/2012/06/carlos_amorales-10.jpg 
La contagion-anaphore, la peur accumulation anaphorique, ou bien cette épidémie interrogative qui harcèle le vers, le temps, l'autre. Le poème multiplie ici les points d'interrogation comme des hameçons du sens, proliférant. Le rapport au réel n'est que questionnement, le rapport à l'autre, questionnement brouillé, échange in abstentia, téléphonique ou non. L'aspect graphique du texte dit une saturation fragmentaire : obsession, répétition chronique, pathologie de la ponctuation qui suggère l'incertain, le quoi, le pourquoi, l'irrésolu. Le poème étale la folie interrogative que suscite la performance artistique, la logorrhée qui loin de freiner l'angoisse, l'incarne et la relance sans cesse en précipitant le rythme de ses mots. Le poème sursaute continuellement, démantèle, distribue le même dans l'autre à partir d'un univers fini de mots, parfois de monosyllabes où le sens se réduit au son, unité phonique ouverte et minimale. Si l'action est bien au cœur du poème, elle en demeure tronquée, contradictoire, chaotique : « ¿Viniste? ¿Te fuiste? / ¿Huiste? ¿Llegaste?».

Dans cette chaîne interrogative pourtant un vers se répète, irradiant « ¿qué fue ese ruido? ». Après les enjambements des vers 4-5, 7-8, 14-15, et 20-21 qui le fragmentent diversement, il apparaît dans son unité sémantique au vers 27 . C'est l'interaction du réel qui se fait une place dans le poème, l'imposition des crissements du dehors. Le vers s'inquiète en déplaçant sa coupure, et inquiète alors la relation je/tu, selon un processus d'intensification : invasion plus perceptible de la peur qui fait inlassablement revenir la même question. Le poème se donne comme corps-parlant, tension de la performance sur le papier ; poème panique, où la peur virulente s'inscrit comme parasitage du discours, déconstruit par des strates de questions qui finissent par dire la peur, la peur de la peur.

La seule vraie réponse explicitement donnée est poétique, c'est le travail sur le rythme : l'isolement, la précipitation, la trivialisation du poème par ses verbes et ses pronoms interrogatifs, l'isolement verbal du sujet soumis aux secousses d'un univers difficilement compréhensible. Au bout, après la sédimentation de la quête de sens qui a en partie désarticulé l'intime, il ne reste en effet que le « quoi ».

Ces pages constituent à elles seules la marque tangible d'une contagion parce qu'elles transforment la représentation (performance) en autre représentation (poème), conservant théâtralité, dimension dialogique même altérée, fonds esthésique. C'est bien cette qualité épidémiologique de la peur - à la fois macro-phénomène collectif, grand symptôme social omniprésent, et micro-phénomène individuel, recroquevillement, paranoïa -, une peur entretenue, relayée, infiltrée partout (presse à sensation, unes sanglantes, journaux télévisés, documentaires, chroniques judiciaires, radio, réseaux sociaux, etc.), qui produit sans cesse des répliques ${ }^{12}$, dans le corps, l'imaginaire des êtres, et sur la page. Le poème s'inscrit ainsi dans une chaîne de représentations qui naissent à partir d'un terrifiant "savoir d'arrière-fond $»^{13}$ - disparitions, fosses communes, corps démembrés, impunité - et jouent à leur tour sur la récurrence et la transmission, sur l'extériorisation de l'obsession en présences, comme formes d'exploration psychologique et sociale, comme résistances. Face au pandémonium de l'immédiat, monstruosité postmoderne où on liquide à tour de bras des êtres qui deviennent des corps-objets anonymes, entre vision dantesque et

11 Luis Felipe Fabre, op. cit., p. 26. Documentation de performance de Vestandpage, love is love and fear is fear. everything else is everything else, sur https://proyectoliquido2012.files.wordpress.com/2012/06/vestandpage-17.jpg

12 En référence au fonctionnement de l'épidémiologie des représentations théorisée par Dan Sperber dans $L a$ Contagion des idées. Théorie naturaliste de la culture, Paris : Éditions Odile Jacob, 1996, et aux macro-phénomènes culturels expliqués par l'effet combiné de processus intra-subjectifs et inter-subjectifs, à l'existence de chaînes causales qui vont des représentations mentales aux productions publiques et réciproquement. . 
miltonienne, l'écriture comme vecteur de contagion s'empare de l'enfer pour le domestiquer, le soumettre à son tour aux démons de la poésie : rythmes, voix, vers, travers, traversée.

\section{Zombies, pandémie et anamnèse}

La prolifération des zombies, tour à tour " plaga zombi », " contagio zombi ", " horda de zombis hambrientos $\rangle^{14}$, constitue pour Luis Felipe Fabre une interprétation du Mexique contemporain, où perce la terreur. Son recueil met en circulation une galerie de portraits et de cauchemars - dépouilles errantes et fanatiques, femmes-vampires, figures spiritistes, muses étranges, et surtout foule anonyme de zombies - et se place ainsi dans la filiation mémorielle d'un imaginaire de l'horreur où règnent des figures tutélaires déjà mentionnées. Il déplace ainsi le territoire de la poésie vers un espace de croyances et de perceptions intuitives, où les vers assument leur condition d'oripeaux, imposant à la fois représentation esthétique du chaos et projet po-éthique. Car enfin la foule des zombies qui colonise les pages de la troisième section du recueil « Notas en torno a la catástrofe zombi » (qui en est aussi la section centrale), vient affirmer un au-delà de la mort comme espace de résistance, de mémoire. Face à la lèse-humanité qui les a projetés dans le dernier cercle du pandémonium, les zombies reviennent hanter notre monde, notre imaginaire, notre propension à l'oubli, à l'inertie, par ce déni de mortalité, cet outrepassement des limites de leur propre corporéité altérée, « una jauría de podredumbres », « crispada », « putrefact[a] $»^{15}$, mais insurgée.

La section se compose de 11 poèmes qui sont présentés sans titre ni ordonnancement numérique (qui faisait des poèmes-performances précédents une série de déclinaisons thématiques de la peur), à l'exception d'une petite musique de nuit grinçante « Opening song » qui plante son nouveau décor de cimetière. Très vite une main sort de terre, aidée par l'étrange mouvement d'une syntaxe qui accompagne à l'inverse ce soulèvement. Les vers descendent en effet par palier de pyramide au plus profond d'une tranchée, laissant surgir cette main qui pourrait être le tenochtli ${ }^{16}$ de la fondation originelle :

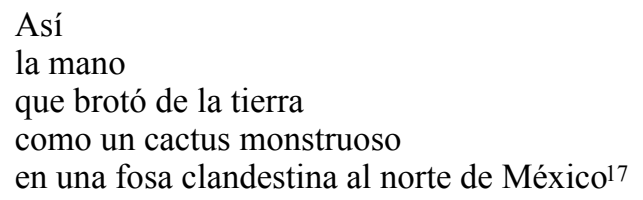

C'est le poème inaugural de la catastrophe zombie, l'endroit où s'origine la pandémie, la terre profanée des premiers temps du monde Mexica. De lieu de vie civilisateur, matière matrice d'un initium culturel, cette terre est devenue abysse, « fosa clandestina », inframonde mortifère, endroit

14 Luis Felipe Fabre, op. cit., respectivement p. 46, 47 et 51 pour les trois citations.

15 Idem, respectivement p. 39, 37 et 38 pour les trois citations.

16 Nous renvoyons au mythe de la fondation de Mexico Tenochtitlan : «Allí donde vino a caer, y habéis visto entre los peñascos, en aquella cueva entre cañas y juncias. ¡Del corazón de Cópil ha brotado ese nopal salvaje! ¡Y allí esperaremos y allí reinaremos! », in Crónica Mexicayotl, « El corazón de Cópil », Folios 86-87, consultable sur http://www.sup-infor.com/sources /liste-nahuatl.htm.

17 Luis Felipe Fabre, Idem, p. 37. 
d'une déportation «al norte de México », d'une défiguration ${ }^{18}$.

A la première main qui se dégage de ses décombres, succèdent des corps entiers, zombies qui s'imposent dans des saisies définitives :

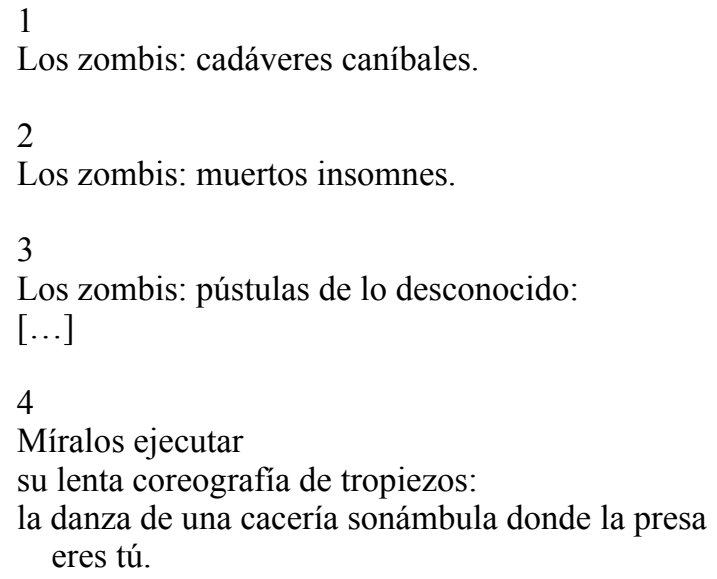

Et ce sont des zombis en marche, qui gagnent du terrain à l'image du vers qui avance puis isole sa victime. La matière poétique joue sur la redondance de syntagmes nominaux qui font aussi état de la contagion : « cadáveres caníbales », « muertos insomnes » l'un contenant l'autre, l'un reproduit dans l'autre, des termes qui trébuchent, se contractent et pétrifient. Ces proparoxytons ont un effet poétique et politique : redoublant l'effroi dans l'homophonie relative, dans la semblance du monstrueux qui appelle le monstrueux « cadáveres » / " caníbales ». Ils semblent gagner ainsi une dimension charnelle, redoublements appétents de son et de sens. Par l'allitération (en [c], [d], [n] et [m] [s]) et l'assonance (en [a], [e] et [es], [o]), ils projettent une manducation, semblent mangés par l'écho des syllabes, et manger à leur tour notre imaginaire, ils s'imposent nécrophages. Ajoutons que ces « muertos insomnes », privés de repos, en rappellent d'autres, contemporains et tout aussi rebelles : des morts incómodos pour Marcos et Taibo II, et ceux indóciles de Cristina Rivera Garza ${ }^{19}$ qui s'emparent des territoires de la fiction policière d'une part, de la critique d'autre part, pour questionner le même territoire, le Mexique zombie du nécro-pouvoir postmoderne. Les poèmes de Luis Felipe Fabre, de vers en vers, relaient la présence inaugurale et systématique de la contagion :

Zombis en las calles

Zombis en las oficinas

Zombis en el centro comercial

Zombis en el metro

Zombis en los parques

Zombis en las azoteas

Zombis en el departamento de abajo ${ }^{20}$.

En occupant tout l'espace urbain (quand autrefois les morts-vivants erraient dans les

18 La mise en perspective de l'imaginaire aztèque intervient ailleurs dans le recueil où surgissent notamment les divinités Coatlicue, mère de Huitzilopochtli, déesse de la vie et de la mort et symbole de fertilité, ainsi que Mictlantecuhtli, puissant dieu de la mort et divinité des enfers (Mictlán), exigeante figure poussant au sacrifice. Pour autant le déplacement de l'horizon à l'univers pré-hispanique n'équivaut pas à proposer une filiation de la violence, tout juste à l'interroger de manière ponctuelle (p. 43), comme il interroge la vierge de Guadalupe, la « Santa Muerte », et des formes contaminées d'un « jesús-maíz transgénico ». Plus vraisemblablement, le poème dit une altération des codes anciens, une corruption des systèmes magico-religieux, une traversée privée de transcendance.

19 Nous faisons référence au roman de Subcomandante Marcos et Paco Taibo II, Muertos incómodos, falta lo que falta, México : Joaquín Mortiz, 2005, et à l'essai critique de Cristina Rivera Garza, Los muertos indóciles, México : Tusquets, 2013.

20 Luis Felipe Fabre, op. cit., p. 47. 
campagnes de Luvina, de Comala), ils marquent aussi le temps, ils deviennent les métronomes d'un présent circonscrit à leur existence : "Zom-bis », la pulsation intime et régulière du monde. Irradiant tout l'espace du vivant, sans limites, ils colonisent indistinctement le matériel. La rose suce l'eau dormante et en meurt. Dans le présent de la décomposition, subsiste quelque chose du passé, de ce qui fut en vie, et cela est projeté par un système de correspondances baudelairiennes - on pensera inévitablement à « La Charogne »- dans une mort active, effervescente. Au cours de ce processus chimique, l'eau putride du vase gagne une animalité, devient « pecera », et contamine la fleur en bouche carnivore : «plantas resucitando carnívoras: flores / zombis $»^{21}$. Tout est donc contagion, susceptible de métamorphoses : matériel, idéel aussi quand la pandémie zombie s'empare de l'invisible, de la rumeur. Le poème alors fait courir le bruit de l'insurrection des morts, fracturant le silence rigide du pouvoir politique, le cynisme, l'irresponsabilité :

Dicen

que los zombis

son una estrategia del narco

para aterrorizar al gobierno. Dicen que

los zombis son una estrategia del gobierno para aterrorizar

a la población. Dicen que los zombis son una estrategia

de la población para aterrorizar al narco. Dicen

que los zombis son una estrategia del gobierno

para aterrorizar al gobierno. Dicen

[...]

Infórmate: escucha Radio Mictlán:

transmitiendo

en vivo

la insurrección de los muertos 22 .

Les deux premières strophes déclinent un univers éteint de mots, qui se déplacent pour trouver du sens mais se referment sur les mêmes formes absurdes. Au-delà de la projection d'un « aterrorizar », et d'une mort « estrategia », tous deux irradiant seuls sur le vers, le reste de la trame perd toute substance vitale, tout potentiel de transformation du monde, en recomposant des équations qui tournent à vide, en s'enfermant dans une rumeur doxique où les acteurs sont interchangeables. Tours de passe-passe, ces constructions formelles font de l'ellipse la manière de se frotter au monde, ou de l'éviter, une attente, un factice jeu de rôles. Aussi la dernière strophe du poème s'impose-t-elle comme un coup de pied dans l'édifice rhétorique antérieur. De la rumeur indistincte et globale du « dicen », le poème préfère le tu et la secousse d'une information émanant des zombies eux-mêmes. La circulation des voix « en vivo » (d'ailleurs placé en promontoire sur le blanc typographique), refonde le poème comme authentique espace de circulation et de résonance. Et le jeu de mots qui frôle l'oxymore, « transmitiendo / en vivo // la insurrección de los muertos », fait chanceler les dernières contradictions entre vie et mort et permet la juxtaposition anachronique et symbolique de « Radio Mictlán », autant de petits séismes contre l'anchylose des vivants.

1

"Todos somos zombis": proclaman

camisetas, grafitis, esténciles, pancartas.

21 Idem, p. 68-70.

22 Ibid., p. 45. 
5

Una ventana: del otro lado del cristal un zombi acecha:

ah, la vieja fábula del monstruo en el espejo 23 .

$\mathrm{Au}$ dernier temps de l'exploration de la catastrophe, apparaît une humanité syncrétique, devenue zombie. C'est l'avènement du tout zombie, du moi-zombie. Des zombies qui incarnent le retour des morts comme la liquéfaction des vivants, dans leur transparence anémique, leur inertie vaguement coupable face à la submersion de violence. Le réseau zombie s'expose, se vend, se revendique. L'asyndète le dit aussi, qui s'étend, recouvre espace intime et espace public, se dissémine au deuxième vers " camisetas, grafitis, esténciles, pancartas » : le réseau zombie épuise le monde dans la prolifération de ses représentations, de ses cris. C'est le fantasme du tout zombie qui inclut donc aussi le reflet du miroir : contagion du regard mais surtout moteur d'une introspection-rétrospection - incorporation de l'autre, rétro-alimentation - retour du revenant et évidement maximal de la substance intime, altérité invasive jusqu'à supplanter. Le fantasme de la disparition, de la métamorphose monstrueuse de l'être laisse penser la latence de l'inerte dans le vivant et du vivant dans l'inerte; l'être n'est plus que latence de mort.

Cette liquéfaction de la présence mondaine pousse la section poétique dans ses retranchements : la pandémie devient inconsistance fantomatique, dimension symbolique d'une post-modernité qui se désagrège, s'évanouit. Particulièrement saisissante est l'image, tirée d'un autre poème de la section, d'une « bolsa vacía, blanca, de plástico. / Una bolsa de supermercado / con la que el viento juega a los fantasmas $\gg^{24}$, figure spectrale enflée par un courant d'air tout rulfien. La poche blanche devient apparition, nuage épiphanique qui pousse à une élévation :

una bolsa

vacía y levitada como el corazón de un santo:

aleluya, aleluya 25 .

Du dessous de la terre au dessus du monde, le parcours assumé du poème, et du recueil, montre une ascension louée exemplairement au dernier vers. Cette spectralisation de la matière inerte sert pourtant bien une mise en perspective. En jeu rien de moins que la trivialisation du sacré - ailleurs aussi rayonnante «Zombi: ¿quién te dijo "levántate y anda"?26 -, la sacralisation du trivial portée à son comble : la poche plastique, à peine enveloppe charnelle encore d'un monde qui s'est dérobé, gagne dans sa lévitation une envergure symbolique. Le flottement spectral du réel qu'elle incarne, substitue bien le néant au sacré. La post-modernité qui règne sur des décombres ne trouve alors qu'à se désincarner et qu'à s'élever au-dessus de son temps et de son espace contaminés, en soufflant ses déchets, ses oripeaux. L'extrême contemporain se livre alors dans sa propre monstruosité.

\section{Nécro-écriture, excavations, poésie-zombie Éléments pour une conclusion}

23 Premiers et derniers vers du dernier poème de la section « Notas en torno a la catástrofe zombi », ibid., p. 56-57.

24 Ibid., p. 53.

25 Ibid.

26 Ibid., p. 41. 
Au seuil encore de Poemas de terror y de misterio, après une couverture-objet qui cache une femme-pieuvre, après des doubles pages où les monstres apparaissent en ombres chinoises, l'oeil touche l'épigraphe du très sombre écrivain américain Dennis Cooper ${ }^{27}$. Cette épigraphe qui intronise la télévision (écran où gesticulent les morts) comme référence cognitive, constitue une anticipation très claire $d u$ glissement $d u$ recueil de Luis Felipe Fabre loin du territoire communément habité par le poème. «Lo único que hacen [los muertos] es pegarte un susto y sonar como a poesía »: l'épigraphe articule justement peur et poésie, imbriquant leur potentiel hermétisme respectif, la sidération ou le rejet qu'elles peuvent générer, et aussi leur étrange musicalité commune, celle du « sonar como » qui contient des échos, des murmures, des cris, tous les bruits du monde en proie à la guerre et au déni d'humanité (enlèvement, torture, exécution sommaire, creusement de la terre, recouvrement). Dans ce "sonar como a poesía ", cacophonie sous-entendue, dissonance-dissidence, on entendra peut-être le grincement $\mathrm{du}$ " crujir de la mariposa », ou un " pío pío » gelmanien, résistant à la mort même du tío Juan, c'est-à-dire des syntagme et onomatopée poétiques qui projettent de la monstruosité dans le poème. On entendra aussi, signalons-le enfin, une propension à l'humour, à l'ironie parfois, propension d'un recueil tout entier qui prend plaisir à se réinventer dans des formes marginales. Si dès lors la poésie est mise en route de la terreur, elle attise aussi ses obsessions et crée ses monstres. Ce postulat, réfracté par l'épigraphe, implique plusieurs conséquences visibles dans l'écriture de Poemas de terror y de misterio.

Une désacralisation du poème, disséminée dans le travail formel

Les titres des sections poétiques et de quelques poèmes du recueil - « Avances », « Breve registro... », « Notas en torno a », « Infomercial », « Trailer 1 », « una ponencia », « Mashup 3 », manifestent la friction à l'ordinaire de l'écriture, friction à la violence délirante - « experiencias escalofriantes », « catástrofe zombi »-, à la possibilité d'un dire, d'un faire de la liberté des mots, un hors-champ poétique. De là, les slogans, les soliloques, les touches conversationnelles, les bandes-annonces de la technoculture de masse, les citations intertextuelles aussi - on pensera au « Desdichado » de Nerval largement déployé - de là, donc, la flexibilité du texte, son aptitude à incorporer à l'écriture le corps impur des monstres. Les effets visuels de la ponctuation miment aussi des focalisations singulières : mouvements latéraux et perpétuels des deux points susceptibles de convoquer toujours une plus-value sémantique, deux points au fond dévorateurs d'espace ; isolement typographique du gros plan aussi, un cri, une main, une chaussure manquante ; tout un tissu de sens qui dispose une raison graphique vouée à perturber le poème, à inaugurer sa nouvelle territorialisation.

\section{Des pages-contagion}

Les poèmes du recueil font de l'écriture le lieu de la migration zombie : transport de revenants qui sont aussi des mots, des sensations repris de poème en poème, traversant les sections poétiques de part en part, véritables syntagmes condamnés à l'errance, et condamnant à l'horreur. Ainsi l'absence contaminante d'une "silla vacía » surgit dans l'obscurité du premier poème «Caminar con los ojos cerrados» (vers 19, p. 22) et revient hanter l'obsession interrogative de « ¿Viniste al performance » (vers 9, p. 26), avant de s'affirmer comme image mémorielle « Otra imagen: / una silla vacía: ¿recuerdas? " (vers 13 puis 14 et 15, p. 30). Il en est de même des « vidrios rotos » propices à la récurrence du fracas initialement présent dans le poème « ¿Viniste al performance ", et qui revient dans les cinquième et sixième poèmes de la deuxième section (vers 2 et 14, p. 28 et vers 5, p. 31). Et encore des « mariposas negras », « un timbre » incongru dont la

27 Voir par exemple http://www.dennis-cooper.net/. 
présence filée - résonance picturale et acoustique - diffuse l'inquiétude, la change en obsession. Cette migration de fragments décèle quelque chose qui rampe dans la diagonale du recueil, de corps poétique en corps poétique, inclus ici et là, là et ailleurs, dans une mouvance élastique qui s'accommode du divers pour déporter les limites de son monde.

\section{Une poésie-zombie}

Le recueil superpose, juxtapose, il réécrit ou désécrit selon la proposition même de son auteur $^{28}$. Il travaille la pandémie zombie dans la mort prolixe de l'extrême-contemporain, la caricature, la défigure encore dans les silhouettes de morts-vivants décidés à refaire surface. Mais cette pandémie zombie pourtant, et c'est aussi sa qualité paradoxale, trace un combat mémoriel, une résistance à la disparition des anonymes sacrifiés, foule vacante transportable dans les mots. Foncièrement palimpseste, le recueil assemble les restes, tatoue la page à l'orée du mauvais goût, se débarrassant par là-même des freins d'une poésie majuscule. Il assemble les restes comme les zombies eux-mêmes affichent un corps où transparaît un autre corps, antérieur, mutilé, trimbalant ce qu'il reste. La désacralisation poétique permet la métamorphose du poème en " thriller hermenéutico $\aleph^{29}$, en espace dégradé, suaire d'une déchirure, et pose, en même temps que la question du genre et de ses limites intensément présente tout au long du recueil ${ }^{30}$, les enjeux politiques de l'écriture dans le contexte mexicain.

¿Qué significa escribir hoy en ese contexto? ¿Qué tipo de retos enfrenta el ejercicio de la escritura en un medio donde la precariedad del trabajo y la muerte horrísona constituyen la materia de todos los días? iCuáles son los diálogos estéticos y éticos a los que nos avienta el hecho de escribir, literalmente, rodeados de muertos?31

Des questions en perspective critique à ces Poemas de terror y de misterio. Et une réponse : « Porque el terror se detiene ahí donde se detiene, inscrita, la palabra terror ${ }^{32}$. Face à l'afflux des morts, à la « production de cadavres comme ultime processus de fabrication de l'économie planétaire $»^{33}$, l'écriture impose celle des mots. Écrire, c'est échapper à la paralysie de la terreur, au terrorisme de l'horreur, horrorisme sans mesure pour Adriana Cavarero. Ce n'est pas tant la mort qui définit la nécro-écriture ${ }^{34}$ que sa capacité à maintenir un rapport entre les vivants et les morts, c'està-dire à penser la littérature comme un dialogue infini, une source-anamnèse d'excavations multiples et de reprises d'une parole qui ne serait plus exclusivement l'apanage des vivants. Poésiezombie alors ces poemas de terror y de misterio où perdure un dialogue entre vivants et morts. Poésie-zombie qui interroge le sort de la condition humaine en questionnant celui du langage : ultime désacralisation de l'auteur, de l'histoire littéraire, de la citation par excellence. Avec la propulsion de Sor Juana dans le monde kitch de la série B, qui occupe la dernière section du recueil «Sor Juana y otros monstruos »- une Sor Juana de fait en prise avec la monstruosité des

28 Luis Felipe Fabre, Leyendo agujeros: Ensayos sobre desescritura, antiescritura y no escritura, México : Conaculta, 2005.

29 Luis Felipe Fabre, Poemas de terror y de misterio, op. cit., « Trailer 2 », p. 17.

30 Pour exemples de la perspective métapoétique dans le recueil, op. cit. p. 22, 24-25, 51, 71, 77 et 93 notamment.

31 Cristina Rivera Garza, Los muertos indóciles, México : Tusquets, 2013, p. 19.

32 Cristina Rivera Garza, Dolerse. Textos desde un país herido, Oaxaca : Sur+Ediciones, 2011, p. 141.

33 Françoise Dastur, «La mort », in Réunions philosophiques 2003-2004, Cannes : Arte Filosofia, p. 22

34 Le terme revient à Cristina Rivera Garza. 
sorjuanistes et avec sa propre monstruosité, tour à tour oiseau rare de la création, Phénix, Énigme et Sphinx -, Luis Felipe Fabre démystifie exemplairement la figure poétique et en repense les démons : la voix auctoriale individualisée est abandonnée pour devenir plurielle et dialogique (« las peores de todas »), la réplique mimétique des univers symbolique, stylistique, métrique de la mystique baroque et du cultéranisme donne lieu à une traversée mémorielle et humoristique du poème, la perfection de la plume succombe au corps pelé des chauves-souris, c'est-à-dire à la contagion du silence, dans un effroi qui finit alors, ultime ironie du sort, par avoir raison des vers :

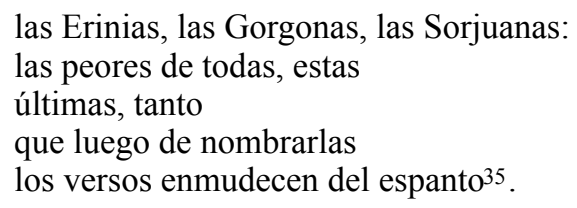

\section{Bibliographie}

Cavarero, Adriana, Horrorismo. Nombrando la violencia contemporánea, Barcelona/México : Anthropos/UAMIztapalapa, 2009.

Derrida, Jacques, La dissémination, Paris : Éditions du Seuil, 1972.

Fabre, Luis Felipe, Poemas de terror y de misterio, Oaxaca : Almadía, 2013.

2005. , Leyendo agujeros: Ensayos sobre desescritura, antiescritura y no escritura, México : Conaculta,

Femández Mallo, Agustín, Postpoesía. Hacia un nuevo paradigma, Barcelona : Anagrama, 2009.

Mbembe, Achille, «Necropolitics», Public Culture, vol. 15, núm. 1, 2003, p. 11-40. , On the Postcolony, Berkeley : University of California Press, 2001.

Negri, Antonio and Michael Hard, Empire, Cambridge : Harvard University Press, 2009.

Perlongher, Néstor, Alambres, Buenos Aires : Último Reino, 1987.

Rancière, Jacques : Le spectateur émancipé, Paris : La Fabrique Éditions, 2008.

Rivera Garza, Cristina, Dolerse, Textos desde un país herido, Oaxaca : Sur Ediciones, 2011. , Los muertos indóciles, México : Tusquets, 2013.

Sperber, Dan, La Contagion des idées. Théorie naturaliste de la culture, Paris : Odile Jacob, 1986.

Subcomandante Marcos et Paco Ignacio Taibo II, Muertos incómodos, falta lo que falta, México : Joaquín Mortiz, 2005 .

\section{Résumé}

Avec Poemas de terror y de misterio (Oaxaca, Almadía, 2013), quelque chose dérape et fait glisser au-delà de lui-même le champ de la poésie : alors sorte de terrain vague volontiers nocturne où se retrouve l'écriture poétique, tendue par les motifs du roman noir, entre fait divers racoleur et épisode de série B. Là, le lieu du poème est un terreau cauchemardesque où surgissent morts vivants et autres visages post-humains dans le rectangle de la page, qui est aussi celui de la pellicule filmique souvent convoquée, ou du trou en terre.

Véritable archéologie de la peur qui fouille les possibilités du texte poétique à ses marges triviales, le recueil met en branle un texte-performance qui dit l'épouvante face à l'irrépressible pandémie de morts du Mexique contemporain. Nous interrogeons ici cette puissance de contagion de la terreur, qui fait de la figure du monstre la seule latence du monde. Une terreur mortifère qui se propage et gagne tout l'espace, espace intime ou tissu social déjà en décomposition, lieu du poème aussi, excavation-palimpseste, réservoir d'une nécro-écriture où irradient les hantises du présent.

Mots clés : poésie contemporaine, Mexique, Luis Felipe Fabre, zombie, nécro-écriture

35 Derniers vers de « Mashup 3 / (Sor Juana y las hermanas murciélago), derniers vers du recueil également, ibid., p. 93. 
Abstract

In Poemas de terror y de misterio (Oaxaca, Almadia, 2013), the poetic field crumbles down, gives away and slips out of its own self - it is no longer a sort of nocturnal wasteland where the poetical writing welcomes the motifs of the Gothic novel, wavering between enticing trivial events and $\mathrm{B}$ series episodes. The place of the poem here becomes in turns a nightmarish ground excavating zombies and other post-human faces from the rectangular page, but also the haunting image of the film reel or the grave.

Acting as an archaeologist of fear digging out the possibilities of the poetical text until its crudest margins are exposed, this collection builds a performance-text voicing the horror of modern Mexico's irrepressible death pandemic. In this article, we intend to enhance such powerfully spreading terror which only preserves the latent figure of the monster. This deadly terror spreads out, invades all spaces - from the intimate realm and the gradually decaying social space, to the place of the poem itself which appears as a palimpsest-excavation and the source of a necro-writing radiating the obsessive fears of the present.

Key words : contemporary poetry, Mexico, Luis Felipe Fabre, zombies, necro-writing 